\title{
Shared Spaces as Authenticity: Exploring the Connectedness of the Physical Environments of Microstreamers and their Audience
}

\author{
Andrew M. Phelps \\ University of Canterbury (and) American University \\ HIT Lab NZ (and) AU Game Center \\ Christchurch, New Zealand / Washington, DC, USA \\ andymphelps@gmail.com \\ Nick D. Bowman \\ Texas Tech University \\ College of Media and Communication \\ Lubbock, Texas, USA \\ nick.bowman@ttu.edu
}

\author{
Mia Consalvo \\ Concordia University \\ Department of Communication Studies \\ Montreal, Quebec, Canada \\ mconsalvo@gmail.com \\ Samuel Smyth \\ American University \\ AU Game Center \\ Washington, DC, USA \\ ss4248a@student.american.edu
}

\begin{abstract}
This work examines how the on-camera environments of small streamers with extremely limited audiences (i.e. microstreamers) generate a form of authenticity directly from the unstaged nature of said environments, and through the multi-purpose nature of these locations. While much of the current research on streaming has focused on larger, more professionalized (and monetized) activity, the microstreams explored here are significant in that they create a very different sense of audience engagement. The combination of (a) the unstaged nature of microstreaming environments, combined with (b) unscripted and unplanned actors and interruptions (pets, other members of the household, etc.) as well as (c) widely varying production values that range from nonexistent to low-budget mimicry of more professionalized streamers, work together to generate a kind of intimacy that is consciously or unconsciously leveraged by the streamer themselves. In their failure to successfully demarcate frontstage and backstage efforts, microstreamers successfully engage audience members in the messiness of life.
\end{abstract}

\section{Introduction}

This paper examines shared space in live streaming, defined as streamers either intentionally or unintentionally sharing their physical spaces with an audience. While much of the research on streaming has focused on larger streams with advertising potential and/or connection to esports phenomena [1][2], there is emerging research on the concept of "microstreaming"-streams whose audiences are often as low as single digits [3] — and their importance as smaller, more intimate spaces. One aspect of these microstreams that is underexplored is the idea of shared space in a physical sense (the invitation of the viewer into the home of the streamer). Given their casual nature, microstreamers are much less likely to have invested in professional level gear such as green screens and lighting rigs, or to have dedicated streamingspecific areas of their homes. Some have argued that intimate spaces such as bedrooms can be considered performative [4][5], but we question the broad applicability of such findings, especially with respect to microstreamers often streaming from intimate spaces as a matter of convenience or necessity. Instead, we argue that microstreams generate a form of intimacy and trust because of their small, familial nature. This is due in part to the seemingly unstaged and shared nature of the environments themselves, which then appear to be leveraged either consciously or unconsciously as a tool for intimacy.

\section{Theoretical Background}

Most investigations of streaming center on the activity and/or people being streamed, but we argue that other artifacts of the streaming event or interaction are as just as critical to understanding streaming engagement and behavior. One such artifact is the physical location of the stream - the space portrayed on camera that the streamer intentionally or unintentionally shares with their audience. Below, we discuss these shared spaces as shared places, and discuss the social demands of sharing one's personal space through streaming [3][4][6][7]. 


\subsection{Shared Spaces as Shared Places}

One way to understand these shared spaces is through the lens of place. For most of us, our social and community engagement happens in "third places" public places defined by their openness and inclusivity (for example, in the ideal "third place" social status markers are less relevant) [8][9]. Third places are distinct from our workplaces ("second place") and our intimate and private homes "first places") [8][9]. From this perspective, streaming represents an event in which the barriers around the "first place" are intentionally removed, and spectatorship invited [5]. Essentially, what is a "third place" to the audiences just casually dropping by to watch is the "first place" for the streamer themselves. Professional streamers navigate this knowingly and intentionally [4] whereas microstreamers may be less aware and/or able to fully control their space. Moreover, professional streamers might make use of more advanced studio technologies (such as green screens) to intentionally mask their home environs or have home studio or office space specifically optimized for streaming (carefully curated to complement streaming activities), whereas microstreamers temporarily repurposing their first place for streaming are far less likely to have the knowledge, resources, or motivations to engage in similar space optimization. Applying Goffman [10], shared spaces by microstreamers can be understood as an unintentional "leaking" of one's privately held backstage, as during the stream itself the microstreamer's "first place" is de facto transformed into the audience's "third place." One implication of this backstream leakage is that it could add an increased perception of credibility to the microstream, as viewers might see the streamed activity as organic to the streaming environment. Of course, such "leakage" could also be understood as an unintentional violation of the streamer's own privacy boundaries [11] in which private, backstage information is made available for consumption by unknown others. Such privacy violations can be somewhat benign (such as seeing evidence of the streamer's other hobbies and interests on their bedroom shelves or living room walls), but others could be more concerning (such as materials identifying their home address, prescription medications or other medical treatment equipment, or other such identifiers).

\subsection{Shared Spaces and Social Demands}

Another way to think about shared spaces is through the lens of interactivity-as-demand [6]. The model can account for our experiences with interactive media (often focused on video games) but has been expanded to other media including streaming [7][12]. This approach suggests that interactive media trigger various demands of the users' limited capacity to process information [13][14], and that these demands cluster around five stable sources: cognitive demands, emotional demands, physical demands (divided further into interface and exertional demands), and social demands. The presence of these demands has been replicated across different cultures and languages including German [15] and Mandarin Chinese [16] and are used to understand how perception of interactive media influences entertainment outcomes.

Of specific relevance to the current study is a focus on social demands - defined as the implicit or explicit awareness of the presence of social others while engaging an interactive medium [6][7]. Originally conceptualized to understand avatars and characters in digital worlds (e.g, in-game characters or other social elements inside a simulation), social demands can also include social others in the environment, both the physical and the online environment. Bowman et al [17] found that playing video games in front of other people was a source of arousal that influenced in-game performance, explained as a social facilitation effect [5][18]. Applied to streaming, social facilitation effects could help facilitate the streamer's performance (i.e., helping them perform better due to the social pressures of being on a digital stage). Scully Blaker et al [19] offer a more comprehensive explanation of the social demands of gameplay through the perspective of tandem play, explaining a common practice of playing singleplayer video games in social environments as a way to share the experience. The authors found that pairs who regularly play this way often privilege sociality over gameplay, using the experience to maintain, explore and/or deepen their relationships - such as those viewing through streams. Extending further, microstreaming can also be understood as a purposeful attempt to increase the social demands of the experience (at least those felt by the performer) by inviting others to the streamed performances (Lin et al., 2019). Given that microstreaming is a mostly hobbyist pursuit (most microstreams are not monetized; Phelps et al. 2021), a social demand perspective might explain why microstreaming could be intrinsically rewarding for the streamer. For example, through the lens of selfdetermination theory [20], game streaming could be a way for streamers to feel an increased sense of relatedness with a live (or curated) audience [21]. For microstreamers, the "payoff" of streaming is less about a fiscal (re: external) motivation and more about an interpersonal (re: intrinsic) payoff. 


\subsection{Sharing Spaces as Authenticating Acts}

Live streaming videogame play on Twitch comprises a diverse set of actions and performances supplied by an equally wide-ranging set of individuals and groups. Yet another way to understand how authenticity is conveyed in live streaming is to consider how these performances are similar to reality television. For example, in their investigation of viewers of reality television, Rose and Woods looked at how individuals interpreted the viewing experience as "a sophisticated quest for authenticity" [33, p. 284]. In their study they argue that authenticity is found via the negotiation of three paradoxical elements, including situation, identification, and production. Most relevant here is their finding that for production, "viewers find themselves seeking balance between the natural narrative and the manipulated narrative, the spontaneous and the scripted, and being and acting" [33, p. 292]. While this may seem antithetical to reading a certain situation as real or authentic, they argue that "the tools of the cinematographer's trade may be used to enhance the reality of what is presented on screen so long as the elements of production are transparent from the viewer's perspective" [33, p. 293]. In other words, microstreamers may not be 'performing' in the same way that reality television characters are, but they are similarly employing a contrived situation (setting up a camera and/or microphone on themselves) while streaming their hobbies, even if they are doing so as hobbyists. To some degree, live streamers are also concerned with the production values of their setup, including their platform, streaming space, and the presence or absence of others - all in an effort to frame the scenes as non-contrived (re: authentic) as possible.

\subsection{Current Study}

The current study represents a preliminary and exploratory attempt to better understand how microstreamers share their environments as part of their live-streamed activities. One suggestion posited elsewhere [3][22] is that microstreaming behaviors might appear more authentic and intimate, as the spaces that house the streaming are temporarily repurposed from the microstreamer's home environment. Given this framing, the research team was engaged in two primary research questions in examining these phenomena. First, we simply wondered whether microstreamers have ways of implicitly or explicitly conveying authenticity:

RQ1: Do microstreamers' shared spaces communicate intimacy and authenticity?
Related to this, and owing to the concept that the amateur or hobbyist nature implied through microstreaming might influence the production value of the streams with possible effects on their sense of being genuine, a second question emerged:

RQ2: How do limited resources impact streams (of microstreamers)?

\section{Methods}

To better understand shared spaces during streaming, we selected a pool of 17 streamers that fit our criteria (i.e., microstreamers who streamed from ostensibly private or intimate spaces) who were predominantly English speaking, and who streamed regularly during the US/Eastern time zone, resulting in a predominantly American and European audience, with a few from Latin America. Some attempts were made to include a range of presumed age and gender, although demographic information was not collected other than what was publicly available on the stream themselves. (e.g., some streamers were obviously parents themselves, while others were college students living at home during the pandemic, etc.) Furthermore, we purposefully selected a variety of different stream types, as microstreaming is not restricted to video game streaming [3][32]. Over the course of 35 weeks during the period August 2020 through May of 2021, we observed these same streamers multiple times per week for periods lasting between roughly 15 minutes and an hour, depending on the availability of the streamer. We compiled screen shots and field notes on prominent objects or scenery in the scene, as well as extraneous interactions such as a pet, parent or sibling wandering through the environment.

\subsection{Sampling Streams}

Streams were initially selected using the criteria above in combination with a now-defunct tool in the Twitch interface, which was the use of the 'sort by (reverse) audience size' option. This tool used to enable users to specifically seek out smaller streams with very limited audiences - an important criterion for defining microstreamers [31]. It was removed by Twitch itself towards the end of the research project in a decision that was controversial among many users [23], well after initial streams for this project had been selected on this basis. Additional microstreams were also found that were derivative from those initially selected, such as those linked by audience members in the initial streams or that were otherwise mentioned by study participants (e.g., during their own streams or in reply to comments). 


\subsection{Field Notes and Coding}

Each week, the research team would revisit the selected streams and add to the field notes for that stream by date, observing actions, scenery, anecdotes, chat behaviors, and more. While each stream was visited, not every streamer went live each week, in which case absences were noted. The observations from the field notes were then coded in a table as to examples that pertained to 1) evidence of successful chat engagement, 2) desire for chat engagement but with little to no observable action, 3) evidence of multipurpose space (i.e. the environment used was not dedicated to streaming alone), 4) evidence of specific actions by the streamer to promote community building, 5) evidence of stream interruptions by others, 6) evidence of seemingly unstaged scenes, and 7) discussion of or practice modified by the COVID-19 pandemic. In addition, each stream was categorized regarding its production value, from essentially nonexistent to clear emulation of professional streaming practices. The research team would meet weekly to discuss observations of each stream as the notes were compiled and encoded. Note that for the discussion and analysis provided here not all these initial codes were relevant (such as some of the chat coding), as they were intended to capture data outside the scope of the research questions for the current study.

\section{Results \& Analysis}

In reviewing the data and notes on these microstreamers, we noted that three major themes emerged from our observations. First, several streamers showed little to no optimization of their spaces for presentation or modification for their audience. These spaces were essentially an unvarnished and unstaged window into their environments. Second, streams also exhibited several instances of unstaged actors or additions to the stream that unintentionally break the fourth wall. From siblings to parents to pets, these spaces are shared not just by the microstreamer, but by their friends and families, and this has several connotations regarding generating authenticity and engagement. And third, we noted that many of these streamers have very low production values, and at times engage in mimicking the production values of more professional streams, but often without budget or resources, in ways that can again generate a sense of authenticity and charm. Each of these themes is discussed in detail in the following sections through case examples drawn from our data set.

\subsection{Shared Unstaged Spaces}

As noted, many microstreamers made little to no effort in staging or customizing their spaces for their audience, conflating their "first place" and their streamed space as one and the same. A streamer named a_potatoe_underscore, for example, used multiple cameras for his VR setup, which also revealed his cluttered and 'lived in' bedroom. a_potatoe_underscore also gave viewers a unique look into his living space compared to other microstreamers by utilizing what he called a "face cam," which was a camera set up that gave the viewer a first-person view from a_potatoe_underscore's perspective (see Figure 1). This unique element not only gave the viewer a look into a_potatoe_underscore's space but allowed the viewer to experience his space the exact way that he would experience it. Another VR streamer, Dni0, had two camera setups; one for when she was playing the rhythm game Beat Saber and one for when she was finished and addressing chat. While the Beat Saber cam showed her physical self in the VR game space, the second cam revealed her room, complete with a messy bookshelf and a crude green screen setup to achieve the effects in the first cam (see Figure 2). Another streamer, FusionMoose, utilized a camera and green screen setup similar to Dni0, though he was playing Minecraft. However, his setup did not account for ambient audio since the viewer could clearly hear an episode of Family Guy on his television in the background. Another streamer that was observed was Yuna_Sakae. Like Dni0 and a_potatoe_underscore, Yuna was playing a dance game, specifically Just Dance 2020. Unlike her fellow dance game streamers, Yuna put her physical space front and center, having most of her stream be taken up by a full body shot of her in her room with the game relegated to a corner of her screen. Though her space was bare, it was not a room optimized for full body streams, with her chair visible as well as items on a table behind where the game screen was on stream. Her stream also doubled as her workout time, for which she dressed in sweatpants, a sports bra, and what looked to be lifting gloves. She also seemed to be streaming from the high level of an apartment building, with the window behind her opened to show the skyline of a city. A streamer named spicenugget streamed during the NaNoWriMo challenge [24] from a home office environment, including a messy bookshelf and a partially dehydrated plant in the background. Spicenugget also streamed the game Hades in the same space, and no change of scenery occurred between the two streams. Another streamer, Streamikitti, had what at first glance looked like a well set up bedroom behind her, including a made bed and a well-organized bookshelf, but on closer examination she also had two 

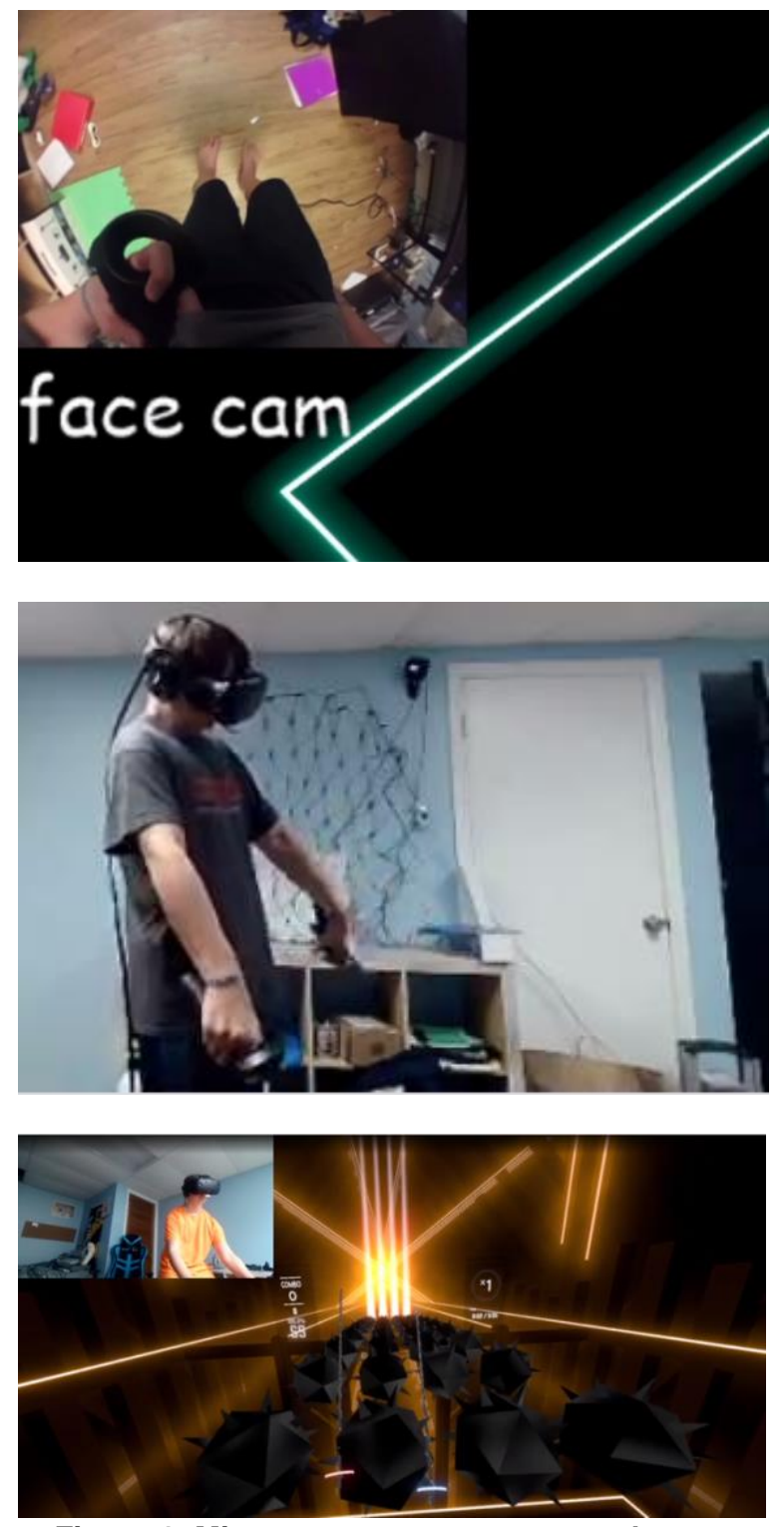

Figure 1: Microstreamer a_potatoe_underscore provides unique views of his environment using a 'face cam' while playing virtual reality game BeatSaber (top) with views of unstaged environment (middle) and the environment and game merged on screen (bottom).

tables, one next to her mostly off camera and one far behind her, with random items strewn about on them. Sandaliadermermelada had a unique camera set up like a_potatoe_underscore because she was streaming through a laptop camera, so every time she adjusted the screen of the laptop, she also adjusted the camera, giving the viewer a different look into her space, and many times just giving the viewer a view of the top half of her head and the ceiling, as well as a stuffed animal off on the side of her screen. To some extent, this could be interpreted through the lens as social demand insofar as
Sandaliadermermelada was compelled to readjust the camera for the perceived audience at home. In all of these microstreams, even when the streamers were using somewhat unique camera angles and production practices, the physical location of the stream was seemingly uncurated.

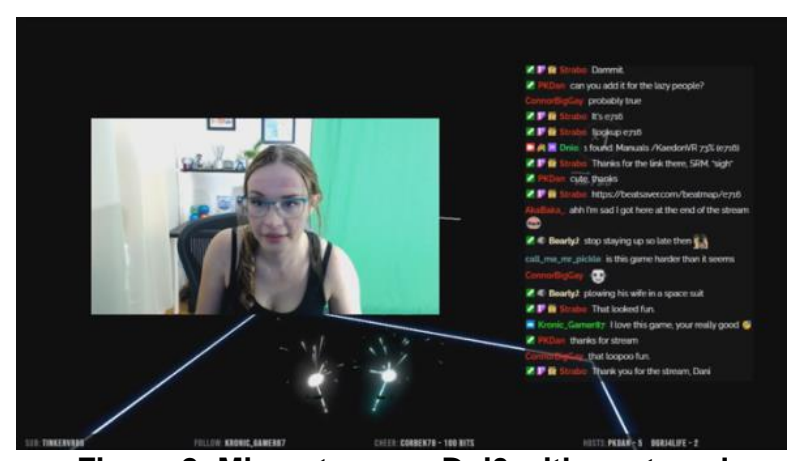

Figure 2: Microstreamer Dni0 with unstaged background and low-production value 'green screen' (sheet) in view.

Some microstreamers consciously leveraged this "first place access" as a means of making their streams more intimate for viewers - showing an intuitive awareness that their "first place" could be a legitimate container or host of the streamed activity. For example, "Dragons in the Dining Room" (DIDR; a live play Dungeons \& Dragons stream) describes their stream as "We are a group of regular people who have come together to play the wonderful game of Dungeon and Dragons and decided to share it with you" as shown in Figure 4. [25] DIDR streams from a nondescript dining room table and uses camera angles and chat functions to reinforce that you (the audience) are a part of this intimate setting. DIDR made efforts to sustain this intimacy even when their players were forced to stream games from separate physical spaces, which was frequently the case due to the Covid-19 pandemic. Some of DIDR's streams have players playing away from the table through Zoom, though each player made sure to have a webcam, and each webcam gives the viewer a glimpse of messy bedrooms and unkempt offices - in a sense, the shared living room was converted into shared private spaces of the individual players, who in turn shared those individual spaces with the viewing audience. Similarly in spicenugget's NaNoWriMo stream, they set a timer for their writing (25 minutes of writing and then a five minute break), and encourage viewers to follow the timer and participate with them, writing during the twenty-five minutes and then sharing what they had written during the five minute break, as shown in Figure 3. Through this, spicenugget created a shared writing space with their viewers, creating a stream where the streamer brought the viewer into their writing space and shared their work with them while 
also allowing the viewer to share back. There is a conscious effort to make the audience a salient part of the stream and thus, NaNoWriMo increases the social demands of her otherwise solitary writing.
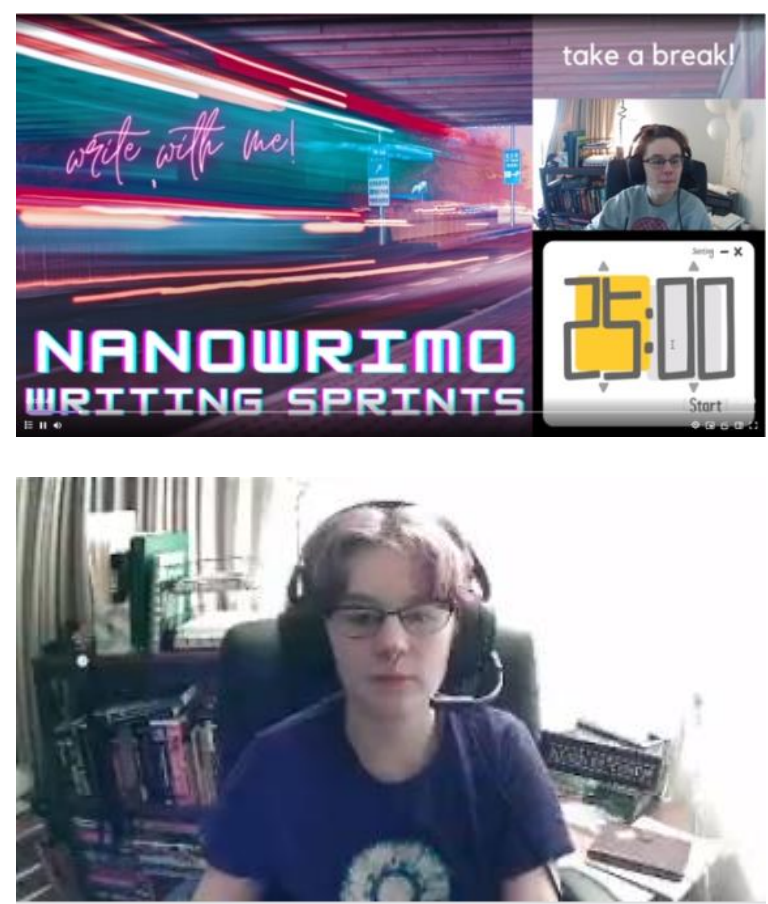

Figure 3: Microstreamer spicenugget streaming during the NaNoWriMo challenge with onscreen timer for audience members to write along (top) and another view of their cluttered, unstaged environment (bottom).

These spaces generated a sense of authenticity because of their unstaged nature and their multipurpose function(s). The spaces being streamed were bona fide first places that were not optimized for presentation to the audience writ large. These practices stand in contrast to larger (and monetized) streamers that often use purposefully dressed sets with items behind them that are trying to grab the attention of viewers or making references to long standing jokes in their communities. For example, Ludwig, a popular streamer on Twitch who averages between 15,000 to 25,000 viewers per stream, has a street sign behind him on stream that reads "Tax Evasion Pog," a reference to a joke within his community about him being a tax evader. Another example is a viral video of wayneradiotv showing viewers what was inside the fridge behind him only to reveal the fridge to be a fake cardboard cutout.

\subsection{Unstaged Actors in Small Spaces}

As noted previously, several streams also exhibited instances of unstaged actors or additions to the stream

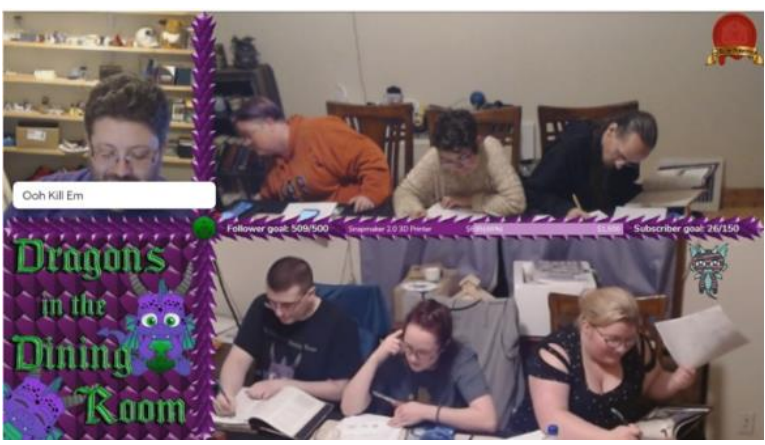

Figure 4: Dragons in the Dining Room, as viewed on Twitch.

that unintentionally break the fourth wall-that presumed barrier between the streamers and audience [26]. Here, we note that while streaming is an intentional breaking of the fourth wall (as streamers open their private spaces to a broad viewing audience), the streaming activity as intentional sharing can be distinguished from entirely unintentional breaches of that space, but with nuance that can result in both intended and unintended consequence. For example, in the DIDR environment, the group gathers around a table where one person's family obviously eats their meals at other times, and there are glimpses of others as they traverse the hallway in the background, often with familial interjections into this shared conversation. These breaches of the fourth wall are compelling, as they remind the viewer that while the gathered streamers are seeking to share their gameplay, the rest of the family (who also has a legitimate claim to the family locations of the streaming space) are unknowingly (and perhaps unwillingly) broadcast to a gathered audience. In another example for DIDR, during one of the gaming sessions a parent wanders through the frame and engages one of the streamers with a request to complete a household chore - a reminder that not only is the shared space one that is co-owned by a larger family unit, but that there is a hierarchy and "livelihood" to that family unit (and by extension, that shared space) that goes beyond DIDR. During one of Streamikitti's streams, she brought her sister in to play a cooperative game with her, though her sister was clearly not in the mindset to stream like she was (see Figure 5), with one exchange leading to Streamikitti mentioning that she was planning on streaming for six plus hours, to which her sister responds that that is "not for her." Streamikitti has also had multiple unplanned interactions on stream, including an interaction where someone she lives with wishes her goodnight during the stream as well as a phone conversation that ended with her saying she could not fulfill a request because "I am streaming." FusionMoose briefly got up from his stream to close his door so a conversation outside his room could no longer 
be heard. a_potatoe_underscore had to pause his stream for a moment to acknowledge a question from his mother whose presence, though off screen, interrupted the flow of his stream (see Figure 6). To some extent, these instances remind us that having unfettered access to a streamer's first space also potentially allows us backstage access, regardless of if that access is in real space or digital space.

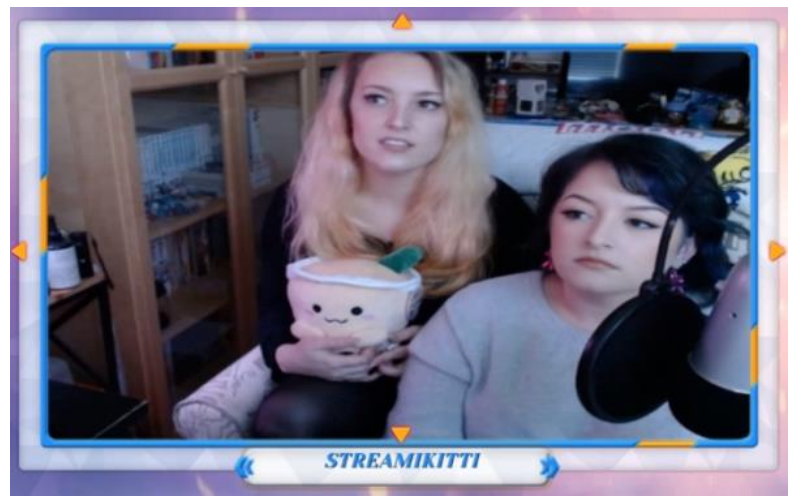

Figure 5: Microstreamer streamikitti (left) with her sister as unscripted on-screen addition.

Several streamers were interrupted by pets during their broadcasts, and in one hilarious example an exercise streamer engaged in an aerobic dance game was being watched by their cat in the window for the entire session. Also, during one of potatoe's "face cam" streams, as mentioned above, he had to chase his cat away from his computer, allowing the viewer to watch the amusing yet also commonplace experience.

Some streamers also merged off-stream and instream interactions. a_potatoe_underscore had one of his friends drop into his stream to continue a prior (offstream) conversation, which dominates the entire stream because of the small audience size. Streamikitti had a moment of chat interaction where she continued conversations on stream that had started off screen. Notably, Streamikitti also would refer to viewers that she knew in real life by their real names, while referring to viewers that she did not know in real life by their Twitch usernames. A British streamer, realpatrick_0, interacted with his two real life friends in chat when they asked him why he streams so late. During one of FusionMoose's streams, he talked with his real-life friend about whether or not he would call his other friend to play Call of Duty.

In these examples, the notion that microstreamers are broadcasting from their first places is reinforced-realworld intimate others and social entanglements cannot simply be "checked at the door" when the cameras are on. These "life interruptions" also speak to a tension for microstreamers with respect to managing content beyond the streamed activity itself, as during live
Salty_carrot it think your mom called you

Pepela

Figure 6: Microstreamer a_potatoe_uncderscore is notified by one of his mods in chat that his mother is trying to reach him.

performance from a lived space there are inevitable disclosures of information and of people who also occupy that space. In contrast, much larger streamers utilize their interruptions to increase viewer engagement and create a more entertaining stream. Streamer timthetatman, a popular Call of Duty: Warzone streamer, frequently has his two year old son appear on stream to engage with chat, having him talk into the microphone directly to his viewers. This led to his son turning off his streaming computer, a situation that timthetatman used as an opportunity to create YouTube video highlighting the moment. The video currently has 2.6 million views [27]. Larger streamers also live with one another and frequently appear on each other streams, both by accident and on purpose, with the streaming house "Offline TV" being a prime example. Finally, any time there has been a serious conversation or an emergency occurring on stream, such as a lost pet, these streamers either mute themselves or turn off their streams entirely. This speaks to the notion that (a) these larger streamers are in some sense borrowing authenticity that microstreamers come by naturally in these contexts, and that (b) in these monetized examples, the 'other actors' are presumably much more aware that they're part of a streaming broadcast, and of its impact.

\subsection{Production Values (or Lack Thereof)}

The third theme we observed is that these streamers engaged in a wide array of camera angles, techniques, and production values likely inspired by more established and professional streamers. While nearly all of the streams would not be confused with more advanced, professionalized efforts, there were drastic differences in how these streamers engaged in their presentation of self within their spaces. a_potatoe_underscore, as noted previously, used multiple cameras to not only show his space but his physical self as he played VR-based games: we see him waving his arms and moving his head while wearing controllers and headgear, and his production decisions are likely informed by de facto standards of other VR streams where showing game footage and body movement are both part of the VR experience. Also, as mentioned above, he was the only VR streamer to provide a unique first-person perspective via his "face cam" set up. 
Dni0 had set up a green screen apparatus, which was literally just a green sheet pulled over one of her walls and some windows. During one of her streams, the sheet came loose, causing her to have to temporarily stop playing and fix it. Another streamer, nickisacoward, spent the beginning of his stream with his "Be Right Back" screen up as he was attempting to fix something off screen. In both this and several other microstreams these graphic bumpers are of variable to poor quality, if they exit at all, with the exception being a few of the art streamers that clearly saw these screens as a way to practice their craft. Nickisacoward then accidentally took the "Be Right Back" screen down, revealing that he was trying to move a fan to a spot in the room so that his microphone would not pick up the noise coming from it. Streamikitti had some of the higher levels of production value that we noted, with the inclusion of pre-stream thumbnails with countdowns to when her stream goes live, to animated emotes that flew across the screen when someone would type that same emote into chat. Streamikitti also had a universal overlay across all the different ways she would stream, whether that was with a full camera displaying her physical space, or a combination of webcam and gameplay footage. However, this high level of production also led to her having production woes. One such repeated issue is her forgetting to switch her capture cards, leading to situations where she has a well set up physically shared space, but viewers cannot actually see the game she is playing. This led to many moments where Streamikitti's high production value would break down and she would admit her inexperience at streaming, especially in reference to the capture card issue, which she acknowledges she has a lot of trouble with.

Of course, not all microstreamers show such an awareness of or interest in production practices. Opposite of Streamikitti is sandaliadermermelada, whose production value is virtually non-existent. Unlike nearly every other microstreamer observed, sandaliadermermelada had to choose between sharing her physical space or her gameplay due to the extremely limited capabilities of her laptop. Viewers watch sandaliadermermelada as she switches between the game she is playing and her webcam, providing the jarring experience of being shifted in and out of her physical space, usually without warning. Another streamer who was streaming the popular game Valorant, also had near non-existent production value, using a camera that was so pixelated you could barely tell he was in his bedroom.

Thus, some microstreamers engage in highly limited production practices, and some seem to actively and purposefully mimic other streams in ways that are variably effective at optimizing the stream itself. Although these production practices have tangible benefits when engaged with precision, their low-fidelity mimicry is more representative of the microstreamer's held institutional logic [28] for how streaming ought to be done. Through these practices, microstreamers exhibit awareness of more professionalized aspects, while simultaneously offering a kind of charm and warmth that can feel absent in larger streams that are attempting the aesthetic of the professional news desk, sports anchor, or talk show host.

\section{Authenticity Generation through Microstreaming}

The sharing of lived spaces with their inclusion of unstaged artifacts, animals, and people, and the loose mimicry of professional or monetized streams, suggest a unique flavor of authenticity among microstreamers that distinguishes them from other larger streamers in key ways.

Authenticity has been defined as a consistent performance of self over time, including letting others see key or core elements of one's personality or experience [29]. Yet as Rose and Wood argued, and this research also finds, authenticity is not simply about a lack of production values - it is instead a set of "more complex processes of signification" [p. 292]. Here for example the unplanned appearance of 'backstage' elements on stream, unplanned interruptions by third parties, and the lower production values as compared to more professional streams, can potentially signal to the viewer that the streamer is just like them, "giving viewers the chance to compare and contrast their own lives" with those streaming [p. 284]. Microstreamers' setups and performances can echo more closely the lives of viewers, allowing for a greater sense of familiarity or judgement about what it is 'really like' for most of us to stream videogames. Microstreamers like the ones examined here also offer viewers access to their personal lives in ways that more professional streams may not. The glimpse of a pet, a concerned parent, half of a phone call, or even a messy bed help to do the work of creating a believable persona for the microstreamer, particularly when such elements appear unwanted or unnoticed. After all, who would willingly bring one's mother onto a stream to be nagged by them? Similarly, even the green sheet substituting as a green screen is much more in line with what the average viewer would likely attempt if they started their own stream. The lack of complete control or polish of one's environment then perhaps ironically helps the microstreamer offer the viewer a fuller picture of themselves, fleshing them out not simply as a streamer but as an authentic individual, with a unique and interesting stream. 
This authenticity generation could also be informed through the perspective of tandem play [19][30]. Another reason that microstreaming could feel more intimate and authentic is through the more informal inclusion of others in streams, both planned and unplanned. The group of friends playing as Dungeons \& Dragons as DIDR with their low production values is likely more akin to one's own experience of playing the same game with friends. Spicenugget inviting viewers to write along with them and then share their results during breaks likewise feels more like a friend inviting one to work alongside them, due to both the smallness of the audience and the more informal nature of the streaming setup. Just as Scully-Blaker et al found with beginning streamers, those with small streams more often end up "streaming with" their audiences, rather than "streaming for" them [19]. In that way their streams are more likely to resemble a group of friends playing a game together in a living room, laughing and joking, than the more performance-oriented and often monetized streams of megastreamers, who more often than not have little to no direct interactions with the majority of their audience members given the scale involved.

\subsection{Future Research Directions}

Along with suggestions noted elsewhere in the manuscript, there are compelling areas of future research regarding microstreaming. For example, it is important to note that we did not perform an audience study - that is, we have no data on audience perceptions and thus, our conclusions regarding authenticity are suppositions based on our own perceptions of the microstreams coded, rather than a larger set of audience response data. For this initial study, we did not feel that we had enough of a sense of what was happening in these microstreams with any sense of regularity in order to effectively frame a reasonable audience survey. Thus, we instead chose first to observe these streams ourselves, to take notes, and to compare the notes of streams against one another to discern the potential patterns and commonalities between them. That said, future research should more directly assess audience perceptions, which could be done using a variety of primary (e.g., surveys, experiments, and interviews) and secondary (e.g., chat analysis and audience coding) data analysis approaches. Interviews with microstreamers themselves would also reveal more relevant data, such as perceived social demands that might result from broadcasting one's hobby compared to engaging it without an assembled online audience (be it synchronous or asynchronous). We would also encourage research into the continued platformization that Twitch and other similar platforms impose on these interactions.

\section{Conclusion}

Microstreamers are a sizable segment of streamers yet tend to be paradoxically overlooked due to their small audiences and non-monetized nature [3][22]. However, they are a unique group of hobbyists precisely for these reasons. The current study more closely considers microstreamers through the lens of shared space - considering both the elements of the spaces displayed on-camera and how those elements might impact how audiences come to understand the experience.

Unlike the overtly performative nature of professional streams [5], microstreamers were observed streaming from their intimate and "lived in" first places, often not manicuring or preparing those spaces for broader public viewing. Streaming from a disheveled home office or messy bedroom adds authenticity to streams already understood in part by their amateur status. For microstreamers, broadcasting from a first place also meant the unintentional broadcast of relational others and through this, private and decontextualized information such as off-stream conversations and other elements of the streamer's "backstage" is streamed as well. These elements, along with the homebrew replication of some professional streaming practices, coalesce to provide a sense of amateur authenticity to microstreamer content.

Finally, the flow of the social interaction between microstreamers, their staged and unstaged human participants and their small audiences do not necessarily blend together harmoniously to create a larger whole, but rather they expose the constructed nature of all streams and the occasional (or even frequent) failure to maintain boundaries between various facets of our lives. In their failure to successfully demarcate frontstage and backstage efforts, microstreamers successfully mimic the messiness of much of daily life.

\section{References}

[1] M. R. Johnson and J. Woodcock. "The impacts of live streaming and Twitch.tv on the video game industry,' Media, Culture \& Society, 41, 5, 670-688. (2019) DOI: https://doi.org/10.1177/0163443718818363

[2] T. L. Taylor. Raising the stakes: e-sports and the professionalization of computer gaming. MIT Press, Cambridge, Mass. (2012)

[3] A. Phelps, M. Consalvo, and N. D. Bowman. "Streaming into the void: An analysis of microstreaming trends and behaviors utilizing a demand framework,' Proceedings of the $54^{\text {th }}$ Hawai'i International Conference on Systems Sciences 
(HICSS), (2021) DOI:

https://doi.org/10.24251/HICSS.2021.349

[4] J-H. Lin., N. Bowman, S-F. Lin, and Y-S. Chen. "Setting the digital state: Defining game streaming as an

entertainment experience," Entertainment Computing,

Elsevier, New York. (2019) DOI:

https://doi.org/10.1016/j.entcom.2019.100309

[5] B. Ruberg and D. Lark. "Livestreaming from the bedroom: Performing intimacy through domestic space on

Twitch," Convergence: The International Journal of Research into New Media Technologies. (2021) DOI: https://doi.org/10.1177/1354856520978324

[6] N. D. Bowman (Ed.). Video games: a medium that demands our attention. Routledge, New York, NY. (2018)

[7] N. D. Bowman. Interactivity as demand: Implications for interactive media entertainment. In C. Klimmt \& P. Vorderer (Eds.), Oxford handbook of media entertainment. Oxford, UK: Oxford University Press (2021).

[8] R. Oldenburg. The great good place: Cafes, coffee shots, bookstores, bars, hair salons and other hangouts at the heart of a community. New York: Paragon House (1989).

[9] C. A. Steinkuehler and D. Williams, "Where everybody knows your (screen) name: Online games as "third places," Journal of Computer-Mediated Communication, 11, 4, 885909. (2006). DOI: https://doi.org/10.1111/j.10836101.2006.00300.x

[10] E. Goffman. The Presentation of Self in Everyday Life. New York: Anchor (1959).

[11] S. Petronio. "Communication boundary management: A theoretical model of managing disclosure of private information between married couples," Communication Theory, 1, 4, 311-335. (2011). DOI:

https://doi.org/10.1111\%2Fj.1468-2885.1991.tb00023.x [12] E. Watts, K. Koban, and N. D. Bowman. "Digital gaming audiences: Awareness, without closeness." Entertainment Computing, 36. (2021). DOI: https://doi.org/10.1111\%2Fj.1468-2885.1991.tb00023.x [13] A. Lang. "The limited capacity model for mediated message processing," Journal of Communication, 50, 1, 4670. (2000). DOI: https://doi.org/10.1111/j.14602466.2000.tb02833.x

[14] J. T. Fisher, R. Huskey, J. R. Keene, and R. Weber (2018). "The limited capacity model for motivated message processing: Looking to the future," Annals of the

International Communication Association, 42, 2, 291-315

(2018). DOI:

https://doi.org/10.1080/23808985.2018.1534551

[15] K. Koban and N. D. Bowman. "Further validation and cross-cultural replication of the Video Game Demand Scale," Journal of Media Psychology, 33, 1, 39-48. (2021). DOI: https://psycnet.apa.org/doi/10.1027/1864-1105/a000280 [16] N. D. Bowman, J-T. Lin, and C. Wu. "A Chineselanguage validation of the video game demand scale (VGDSC): Measuring the cognitive, emotional, physical, and social demands of video games," Proceedings of CHI Conference on Human Factors in Computing Systems. DOI:

https://doi.org/10.1145/3411764.3445348

[17] N. D. Bowman, R. Weber, R. Tamborini, and J. Sherry. "Facilitation game play: How others affect performance at and enjoyment of video games," Media Psychology, 16, 1,
39-64. (2013). DOI:

https://doi.org/10.1080/15213269.2012.742360

[18] R. B. Zajonc. "Social facilitation," Science, 149, 269274. (1965). DOI:

https://psycnet.apa.org/doi/10.1126/science.149.3681.269

[19]. R. Scully-Blaker, J. Begy, M. Consalvo, and S. Ganzon. "Playing along and playing for on Twitch: Livestreaming from tandem play to performance," Proceedings of $50^{\text {th }}$

Hawai'i International Conference on Systems Science.

(2017). DOI: http://doi.org/10.24251/HICSS.2017.246

[20] R. M. Ryan, and E. L. Deci. "Self-determination theory and the facilitation of intrinsic motivation, social

development, and well-being," American Psychologist, 55, 1, 68-78 (2000). DOI: https://doi.org/10.1037/0003-

066X.55.1.68

[21] Q. Zhao, C-D. Chen, H-W. Cheng, \& J-L. Wang.

"Determinants of live streamers' continuance broadcasting intentions on Twitch: A self-determination perspective," Telematics and Informatics, 35, 2, 406-420. DOI:

https://doi.org/10.1016/j.tele.2017.12.018

[22] [ REDACTED FOR BLIND REVIEW ]

[23] r/Twitch - Twich removed the (Low to High) sorting option in Just Chatting. reddit. Retrieved June 11, 2021 from https://www.reddit.com/r/Twitch/comments/i1s5pi/twich_re moved the low to high_sorting_option_in/

[24] NaNoWriMo. Retrieved June 11, 2021 from https://nanowrimo.org/

[25] DragonsInTheDiningRoom. Twitch. Retrieved June 11, 2021 from https://www.twitch.tv/dragonsinthediningroom

[26] T. Brown, Breaking the fourth wall. Edinburgh,

Scotland: Edinburgh University Press (2012).

[27] MY SON SHUT OFF MY PC WHILE PLAYING

WARZONE!! Ft. Nickmercs, CouRageJD \& Cloakzy.

Retrieved June 11, 2021 from

https://www.youtube.com/watch?v=5EjtqaP0qrQ

[28] H. A. Haveman and G. Gualtieri. "Institutional Logics," In R. Aldag (ed.), Oxford Research Encyclopedia of Business and Management (2017). DOI:

https://doi.org/10.1093/acrefore/9780190224851.013.137

[29] Alice E. Marwick. Status Update: Celebrity, Publicity, and Branding in the Social Media Age. Yale University Press. (2013)

[30] M. Consalvo, J. Begy, S. Ganzon, R., and Scully-Blaker. "Tandem play: Theorizing sociality in single-player gameplay." In N. D. Bowman (ed.), Video Games: A Medium that Demands Our Attention. New York: Taylor and Francis. (2018).

[31] Robert Adams. 2019. Twitch Viewers Low to High filter is a big win for small streamers. GameRevolution. Retrieved June 11, 2021 from

https://www.gamerevolution.com/news/558901-twitchviewers-low-to-high-new-filter

[32] A. Phelps and M. Consalvo. Laboring Artists: Art Streaming on the Videogame Platform Twitch. Proceedings of the 53 ${ }^{\text {rd }}$ Hawai'i International Conference on Systems Science. (2020). DOI:

https://doi.org/10.24251/HICSS.2020.326

[33] Randall Rose \& Stacy Wood. Paradox and the Consumption of Authenticity through Reality Television.

Journal of Consumer Research, Vol. 32, pp. 284- 296. (2005) DOI: https://doi.org/10.1086/432238 